\title{
Producing appropriate prosodic cues in a non- dominant language: preliminary results from French-English bilinguals
}

\author{
Annie C. Gilbert ${ }^{1,2}$, Max Wolpert ${ }^{1,3}$, Shanna Kousaie ${ }^{2,4}$, Shari R. Baum ${ }^{1,2}$ \\ ${ }^{1}$ School of Communication Sciences and Disorders, McGill University, Canada \\ ${ }^{2}$ Centre for Research on Brain, Language, and Music, Canada \\ ${ }^{3}$ Integrated Program in Neuroscience, McGill University, Canada \\ ${ }^{4}$ Montreal Neurological Institute and Hospital. McGill University, Canada \\ https://doi.org/10.36505/ExLing-2017/08/0010/000312
}

\begin{abstract}
Adapting one's production of prosodic cues to a second or non-dominant language can be difficult. The present study focuses on French-English bilinguals' ability to adapt their prosody to coordinate phrase-final lengthening and lexical stress. Because French has no lexically-coded prosody, it might be difficult for Frenchdominant speakers to simultaneously control lexical and phrasal prosodic cues. Our preliminary results demonstrate that not only the speaker's L1, but the relative dominance of one language over another can predict speakers' ability to adapt prosody to the specific demands of different languages, at least with respect to controlling syllable duration. These findings are in line with recent results showing that native French listeners do not process lexical stress automatically, instead relying on alternative perceptual mechanisms.
\end{abstract}

Key words: Bilingualism, L2 prosody, stress, lengthening, language dominance

\section{Object of study}

Adapting one's production of prosodic cues to a second or nondominant language can be difficult and may lead to a perceived foreign accent or even render speech hard to interpret. The present study focuses on French-English bilingual speakers' (FEs) ability to adapt their prosody to coordinate phrase-final lengthening and lexical stress. Because French has no lexically-coded prosody, it might be difficult for Frenchdominant speakers to simultaneously control lexical and phrasal prosodic cues. The goal of our study is to examine how FEs with different patterns of language dominance use duration to mark lexical and phrasal prosody in English and French.

\section{Methodology}

\section{Participants}

Nineteen healthy FEs were recruited from the Montréal area. Information about participants' first and second languages (L1 and L2) and language learning history was gathered through questionnaires, and an index of language dominance was obtained by comparing the

ExLing 2017: Proceedings of 8th Tutorial and Research Workshop on Experimental Linguistics, 19-22 June, Heraklion, Crete, Greece 
English and French scores on an adapted sentence recall subtest of the Clinical Evaluation of Language Fundamentals 4 (Semel, et al., 2003).

\section{Stimuli}

The task involved sentence pairs containing the same two-syllable string presented either as two monosyllabic words separated by a phrasal boundary (e.g. English: If you want a key, we can duplicate one. French: Le vendeur d'or loge à l'hôtel.) or as one bisyllabic word preceding a phrasal boundary (e.g. English: If you want a kiwi, I can buy one tomorrow. French: Le vendeur d'horloge vit à l'hôtel.). Thirty sentence pairs were created in each language (English and French).

\section{Procedures}

Sentences (pseudorandomized but blocked by language) were presented on a computer screen, and participants were instructed to read them aloud in a neutral tone. Trials with disfluencies, pauses, or production errors were discarded, along with the matching sentence in the pair.

\section{Analysis}

To control for speech rate variability, relative duration measures were computed by comparing the duration of each syllable of the two-syllable string (S1 e.g.: [ki] and S2 e.g.: [wi]) to the duration of the entire ambiguous region $(\mathrm{S} 1+\mathrm{S} 2$, see Figure 1 for a schematic representation of the measurements). Using these values, we calculated an asymmetry score by subtracting the relative duration of $\mathrm{S} 1$ from the relative duration of $\mathrm{S} 2$ (henceforth "S2-S1 asymmetry"). A positive S2-S1 asymmetry score indicates that the second syllable was relatively longer than the first syllable, and vice versa. All statistical analyses were performed using Generalized Linear Mixed Effects models (Baayen, et al., 2008; implemented in the lme4 package (Bates, et al., 2014), version 1.1-7, in $R$ (R Dev. CoreTeam, 2010), version 3.1.3).

Significance levels were obtained using the lmerTest package (Kuznetsova, et al., 2015), version 2.0-29. This method was chosen over traditional statistical analyses because it takes into account subject-related variability, which represents a great advantage when working with small numbers of participants. Models were fitted with subjects and sentence pairs as random effects (with random intercepts) and language produced (English or French), number of words in the ambiguous region (one bisyllabic word i.e. kiwi -, or two monosyllabic words - i.e. key, we) and participant's language dominance index as fixed factors. 


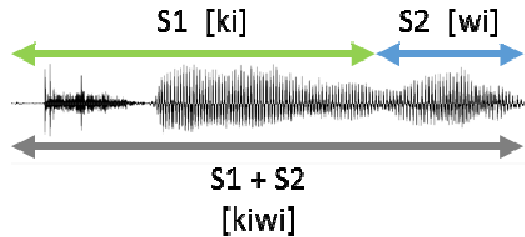

English example

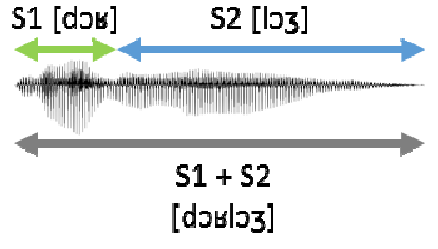

French example

Figure 1. Two examples of S2-S1 asymmetry calculation. Left panel represents an English example with a negative S2-S1 asymmetry score, where S2 is shorter than S1, whereas the right panel represents a French example with a positive S2-S1 asymmetry, where S2 is longer than S1.

\section{Results}

Statistical models revealed a significant three-way interaction among target language (English vs French), number of words in the ambiguous region (1 bisyllabic vs 2 monosyllabic words), and participants' language dominance index in predicting S2-S1 asymmetries $[\beta<-0.07112, S E=0.025, \mathrm{t}=-$ $2.824, \mathrm{p}<0.01$ ] (see Fig. 2). Interestingly, the pattern of S2-S1 asymmetries remained stable across speakers in French trials, but was modulated by participants' language dominance index for English trials. English-dominant and non-English-dominant speakers (balanced bilinguals or Frenchdominant) behaved differently from the English-dominant speakers when speaking in English. In contrast to English-dominant speakers, non-Englishdominant speakers showed a smaller S2-S1 asymmetry within bisyllabic word trials, such that S1 and S2 were approximately equal in duration.

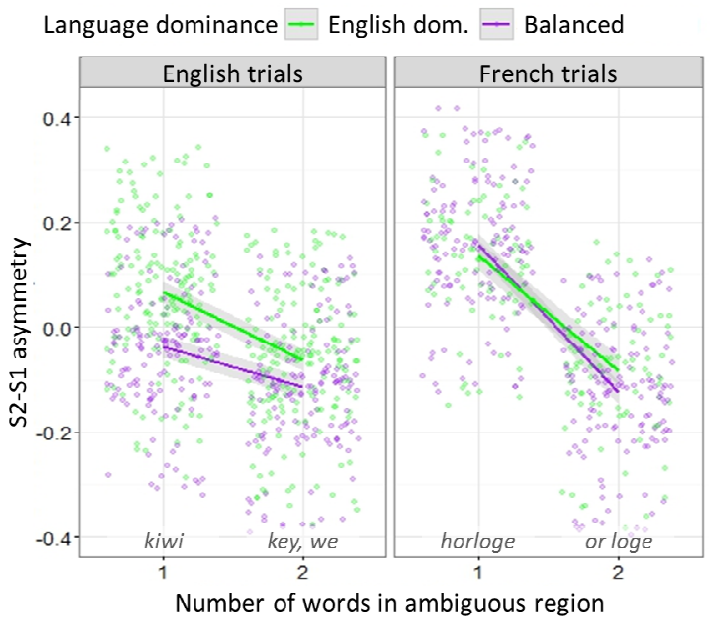

Figure 2. Interaction between the number of words in the ambiguous region ( $\mathrm{x}$ axis), language of the trial (English - left panel, French - right panel) and estimated language dominance (English dominant in green, balanced bilinguals in purple). Fitted data points and linear regressions from the LME model. 
Similar results were obtained if the language dominance index is replaced by the speaker's self-reported L1 $[\beta<-0.1252, S E=0.057, \mathrm{t}=-$ 2.198, $\mathrm{p}<0.03$ ]. That is, only English L1 speakers pattern with English dominant speakers and manage to modulate the duration of the two syllables in the bisyllabic word condition. Non-native English speakers and/or nonEnglish dominant speakers seem to have a difficult time differentiating the duration changes associated with lexical stress from the duration changes associated with phrase final lengthening.

\section{Conclusions}

The preliminary results demonstrate that not only the speaker's L1, but the relative dominance of one language over another can predict speakers' ability to adapt prosody to the specific demands of different languages, at least with respect to controlling syllable duration. These findings are in line with results reported by Michelas et al. (2016), showing that, unlike native English listeners, native French listeners do not process lexical stress automatically, instead relying on alternative perceptual mechanisms.

\section{Acknowledgements}

Funding for this research was provided by the Fonds de Recherche du Québec - Société et Culture, The Blema and Arnold Steinberg Family Foundation, and The Centre for Research on Brain, Language and Music.

\section{References}

Baayen, R. H., Davidson, D. J., \& Bates, D. M. 2008. Mixed-effects modeling with crossed random effects for subjects and items. J. Mem. Lang., 59(4), 390-412.

Bates, D., Maechler, M., Bolker, B., \& Walker, S. 2014. lme4: Linear mixed-effects models using Eigen and S4. (Version R package version 1.1-6). Retrieved from http://CRAN.R-project.org/package=lme4.

Kuznetsova, A., Brockhoff, P. B., \& Christensen, R. H. B. 2015. lmerTest: Tests in Linear Mixed Effects Models. R package version 2.0-29.

Michelas, A., Frauenfelder, U. H., Schön, D., \& Dufour, S. (2016). How deaf are French speakers to stress? JASA, 139(3), 1333-1342.

R Development Core Team. 2010. R: A language and environment for statistical computing. Vienna, Austria: R Foundation for Statistical Computing. Retrieved from www.R-project.org

Semel, E., Wiig, E. H., \& Secord, W. A. 2003. Clinical Evaluation of Language Fundamentals 4 (CELF-4). . San Antonio, TX.: The Psychological Corporation. 